\title{
Valores sociopersonales y problemas de convivencia en la Educación Secundaria
}

\author{
Jesús de la Fuente Arias ${ }^{1}$ \\ Francisco Javier Peralta ${ }^{1,2}$ \\ $\mathbf{y}$ \\ María Dolores Sánchez Roda ${ }^{2}$
}
${ }^{1}$ Departamento Psicología Evolutiva y de la Educación, Universidad de Almería
${ }^{2}$ Orientadores, IES. Almería.

España

jfuente@ual.es 


\section{Resumen}

Introducción. La importancia de la educación en valores en la sociedad actual sigue siendo una de los retos más importantes de nuestro Sistema Educativo. Esta relevancia cobra una dimensión de necesidad inexcusable cuando hablamos de educación en valores sociales durante el período de la adolescencia o la Etapa de la Educación Secundaria Obligatoria (E.S.O.). El estudio, la investigación y la intervención para este tipo de educación se ha centrado, especialmente, en el ámbito de las habilidades y de las competencias sociales (Farrington, 1989, 1993; Tremblay, Kurtz, Masse, Vitaro y Phil, 1995), de tal forma que poseemos numerosos instrumentos y evidencias que sirven para evaluar el nivel de competencia o la inadaptación social de los alumnos.

Sin embargo, el ámbito de los valores de convivencia sigue siendo un terreno por acotar en esta problemática. Para aportar luz a la misma, asumimos los planteamientos de Tierno (1992,1993a, 1993 y y 1996) sobre valores humanos, integrados con el modelo de De la Fuente $(1999,2000,2003)$ que propone trabajar la autorregulación de los valores, a través de sus tres niveles comportamentales constitutivos de los valores, asumiendo que la incorporación de un valor lleva consigo integrar: las cogniciones o pensamientos (sobre lo que una persona piensa o cree en las relaciones interpersonales), los sentimientos o afectos (emociones que asociadas a la vivencia de una situación interpersonal) y las acciones o comportamientos (conductas propias de cada situación interpersonal) propios de ese valor.

Objetivos. A partir estos supuestos nuestro estudio tiene un triple objetivo. En primer lugar, pretende la elaboración y presentación de un nuevo cuestionario sobre valores sociopersonales, analizando sus propiedades psicométricas. En segundo lugar, persigue elaborar un perfil descriptivo de los valores sociopersonales construidos por los alumnos de esta etapa. En tercer lugar, establecemos las relaciones de interdependencia entre el cuestionario sobre valores y otras medidas de comportamientos desadaptativos.

Método. Participaron 857 alumnos y alumnas de dos centros educativos públicos (Institutos de Educación Secundaria Obligatoria) de la provincia de Almería, con un rango de edad entre doce y dieciocho años, de los que 437 sujetos son varones (51.0\% de la muestra) y 420 sujetos son mujeres (49.0\% de la muestra), que se hallan cursando estudios de Educación Secundaria Obligatoria (véase en el cuadro siguiente la distribución por curso). A partir de 
esa muestra hemos realizado tres estudios consecutivos para lograr los tres objetivos propuestos.

Resultados y Conclusiones. El primer estudio nos ha permitido obtener un Cuestionario de Evaluación de Valores Sociopersonales con unos valores psicométricos iniciales aceptables. El segundo estudio muestra una interesante radiografía de los valores sociopersonales de nuestros adolescentes, respecto a otras variables sociodemográficas y académicas de los alumnos. El tercer estudio confirma las relaciones de interdependencia e importancia de los valores sociopersonales y la conducta antisocial.

Palabras Clave: Valores sociopersonales, Convivencia, Adolescencia, Evaluación, Conducta antisocial. 


\section{Introducción}

El área de la conducta prosocial y, su polo opuesto, de la conducta antisocial, han sido objeto de diferentes formas de estudio y de acercamiento a esta problemática. La convivencia en los contextos escolares está presidida por conductas que conforman un amplio abanico, desde las más adaptadas y acordes con lo que se espera de los estudiantes escolar y socialmente, hasta otras, que van en dirección opuesta, y que terminan en el extremo menos deseable. Dentro de las conductas desadaptativas hay que considerar un amplio repertorio conductual con una etiología, sintomatología y pronóstico muy diverso. Todas ellas coinciden en que desestabilizan la convivencia en el aula y en el centro, haciendo más difícil e incluso imposible la consecución de objetivos académicos. Como ya se ha expuesto en otros estudios (Peralta, Sánchez, Trianes y De la Fuente, 2003), estas conductas pueden catalogarse de tres tipos:

a) Desmotivación y desinterés académico provocando desastrosos resultados escolares, afectando gravemente a otros procesos como autoestima, competencia social, identificación con el grupo-clase al que se pertenece, e inadaptación escolar. Ortega y Del Rey (2003) exponen que la desmotivación escolar es, a veces, causa y, a veces, consecuencia de la conflictividad escolar.

b) Conductas disruptivas, que pueden definirse como las que producen ruptura, desestabilización brusca de la convivencia (Fernández, 2001). Entorpecen la marcha de la clase perjudicando gravemente al propio alumno que las ejecuta, como al resto de compañeros y profesores que las padecen. Algunas investigaciones han sugerido que pueden estar asociadas a conductas externalizadas (Kazdin y Buela-Casal, 1996) que son conductas psicopatológicas problemáticas, de diversas intensidades y pronósticos, pudiendo también conducir a la inadaptación escolar.

c) Conducta agresiva, antisocial (Farrington, 1989, 1993; Tremblay, Kurtz, Masse, Vitaro y Phil, 1995), señalada por la investigación como de alto riesgo de padecer problemas posteriores en la adolescencia o en la vida social, al terminar la enseñanza obligatoria. En esta categoría, aunque reconociendo que se trata de una línea de investigación diferente de la anterior, puede introducirse también la conducta agresiva de intimidación a un compañero, estudiada exhaustivamente por la investigación sobre el comportamiento bullying (Olweus, 1993; Ortega, 2000; Smith, Morita, Junger-Tas, Olweus, Catalano y Slee, 1999), desde la perspectiva del agresor, la víctima o los espectadores, así como las consecuencias a largo plazo de vivir estas experiencias de violencia. 
El estudio, la investigación y la intervención para esta problemática se ha centrado, especialmente, en el ámbito de las habilidades y de las competencias sociales (Farrington, 1989, 1993; Tremblay, Kurtz, Masse, Vitaro y Phil, 1995), de tal forma que poseemos numerosos instrumentos y evidencias que sirven para evaluar el nivel de competencia o la inadaptación social de los alumnos.

Sin embargo, el abordaje desde la perspectiva de los valores no ha sido considerada con la misma intensidad, aunque es plausible plantearse que la problemática de la convivencia escolar se encuentra en estrecha relación con los valores que cada individuo le concede a su relación con los demás congéneres que le rodean y al beneficio o provecho que quiere obtener de su relación. Prueba de la escasa constatación de esta relación es la escasez de cuestionarios que evalúen el tipo de valores sociopersonales de los alumnos, especialmente, en la Educación Secundaria. Por ello, entendemos sigue siendo necesario indagar en el tipo de valores que cada persona pone en funcionamiento en su relación con las demás personas de forma que permitan poner en marcha estrategias psicopedagógicas que sirvan no sólo para corregir posibles comportamientos desviados, sino también para fomentar valores prosociales en todos los sujetos escolarizados con objeto de incrementar los valores democráticos, de cooperación y tolerancia.

Desde del área de la orientación y de la acción tutorial se ha constatado la necesidad de implementar programas de evaluación e intervención que permitan mejorar la convivencia en los centros escolares, preparando a los jóvenes para su incorporación a la vida activa y social de forma participativa y responsable. En este contexto, es relevante plantearse la construcción de un nuevo instrumento de evaluación, diseñado para ser contestado por el alumnado, que pueda arrojar luz sobre algunas de las causas que influyen y condicionan el desarrollo de las relaciones interpersonales y que pueden ser originarias de conflictos, al tiempo que permita establecer pautas de intervención para la mejora de la convivencia escolar.

Para aportar luz a la misma, asumimos los planteamientos de Tierno (1992,1993a, 1993b y 1996) sobre valores humanos, integrados con el modelo de De la Fuente (1999, 2000, 2003) que propone trabajar la autorregulación de los valores, a través de sus tres niveles comportamentales constitutivos de los valores, asumiendo que la incorporación de un valor lleva consigo integrar: las cogniciones o pensamientos (sobre lo que una persona piensa o cree en las relaciones interpersonales), los sentimientos o afectos (emociones que asociadas a la vivencia de una situación interpersonal) y las acciones o comportamientos (conductas propias de cada situación interpersonal) propios de ese valor. 
El uso de la recogida de información a través del propio alumno se ha considerado un procedimiento fiable, ya que los autoinformes suministran información importante en temas como el autoconcepto, atribuciones, actitudes y sentimientos. En el mismo sentido, los autoinformes en los que participan adolescentes superan las limitaciones de niños de edades inferiores debido a su madurez y desarrollo sociocognitivo, así como a la interiorización de normas y valores sociales (Trianes, Blanca, Muñoz, García, Cardelle-Elawar e Infante, 2002).

Por todas las consideraciones anteriores, los objetivos de este trabajo fueron:

1) Construir un nuevo instrumento que permita evaluar distintos tipos de valores sociopersonales relacionados con la convivencia en los centros escolares de Educación Secundaria Obligatoria. Ello supone desarrollar todo el proceso de elaboración, prestando especial atención a los análisis de fiabilidad y de validez.

2) Elaborar un perfil descriptivo de los valores sociales que los alumnos de esta etapa tienen incorporados a su escala de valores. En este punto esperamos encontrar que los valores sociopersonales más impulsados por el entorno sociocultural sean los más construidos por los alumnos y viceversa.

3) Establecer las relaciones de interdependencia entre los valores sociopersonales para la Convivencia y otras medidas, ya estandarizadas, sobre los comportamientos sociales inadaptados de los alumnos. Este tipo de análisis puede aportar luz sobre la insuficiente construcción de valores -y no solo de habilidades sociales- que los alumnos de la Educación Secundaria pueden mostrar (De la Fuente, 1999). En este caso, esperamos encontrar que, a mayores valores de convivencia escolar del alumnado, éste puntúe más bajo en conductas de tipo antisocial.

\section{Método}

\section{Participantes}

Participaron 857 alumnos y alumnas de dos centros educativos públicos (Institutos de Educación Secundaria Obligatoria) de la provincia de Almería, con un rango de edad entre doce y dieciocho años, de los que 437 sujetos son varones (51,0\% de la muestra) y 420 sujetos son mujeres (49,0\% de la muestra), que se hallan cursando estudios de Educación Secundaria Obligatoria (véase en el cuadro siguiente la distribución por curso). La media de edad es 
de 14.37, con una desviación típica de 1.33. Todos los sujetos cumplimentaron los instrumentos objeto de estudio o, en su caso, cumplimentados por sus profesores.

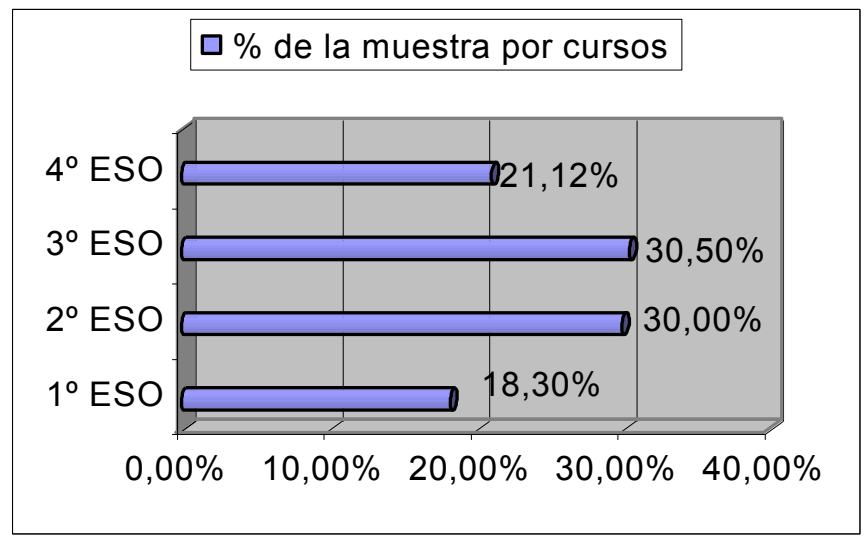

Cuadro 1. Distribución de la muestra por cursos de la ESO.

\section{Estudio 1. Construcción de un cuestionario sobre valores sociopersonales para la conv-} vencia.

\section{Método}

\section{Procedimiento}

Este instrumento fue elaborado, inicialmente, de manera racional, a partir de dos planteamientos previos:

1) La propuesta de valores sociopersonales para la convivencia, extraída a partir de la revisión bibliográfica efectuada sobre valores humanos y convivencia escolar (Bilsky y Schwartz, 1994; Braithwaite, Law, 1985; De la Fuente, 1999; Levy y Guttman, 1974; Markus y Kitayama, 1991; Schmitt, Schuwartz, Steyer y Schmitt, 1993; Schwartz y Sagiv, 1995; Schwartz, 1994; Tierno, 1992, 1993a, 1993b, 1996).

2) La propuesta racional de las tres dimensiones inherentes a cada valor, implícitas en la concepción de la autorregulación de los valores (De la Fuente, 1999, 2000):

a. Pensamientos: Ideas que la persona tiene sobre sí misma; expectativas (autoconcepto); creencias, valores; guiones, esquemas; habilidades de autorregulación y autocontrol de este nivel.

b. Sentimientos: Emociones y afectos ante sí mismo (autoestima), sobre los demás y el 
mundo; comportamientos afectivos; habilidades de autorregulación y autocontrol de este nivel.

c. Acciones manifiestas: nivel de ejecución de conductas, habilidades o destrezas personales e interpersonales; habilidades de autorregulación y autocontrol de este nivel.

El instrumento inicialmente surgido es la versión racional del Cuestionario sobre Valores Sociopersonales para la Convivencia, que evalúa cogniciones, emociones y conductas relacionadas con la integración y la convivencia escolar. Consta de 93 items, con respuesta en cinco escalones («Muy en desacuerdo», «En desacuerdo», «Regular de acuerdo», «Bastante de acuerdo» y «Muy de acuerdo»), que el alumnado de cada curso pudo cumplimentar. La cumplimentación por parte de los alumnos, siempre de forma voluntaria en situación de grupo de clase y en horario lectivo. La recogida de datos se efectuó entre los meses de enero y junio de 2003.

\section{Análisis estadísticos}

Efectuamos los análisis pertinentes para constatar la validez y fiabilidad del instrumento. En el primer caso, los análisis factoriales exploratorios de primer y segundo orden, a través del Método de Componentes Principales, Oblimin con Kaiser, proporcionaron la estructura factorial del instrumento. En el segundo, la fiabilidad fue obtenida a través de la prueba Alpha de Cronbrach.

Para el tratamiento estadístico se ha utilizado el programa informático estadístico SPSS (1999), versión 10.00.

\section{Resultados}

\section{Análisis factorial exploratorio}

La medida de la adecuación muestral de Kaiser-Meyer-Olkin (KMO) fue de 0.97 y el test de esfericidad de Bartlett $\left(\chi_{2}=41395.159 ; \mathrm{p}=0.000\right)$ fue estadísticamente significativo. El análisis de componentes principales, rotación Oblimin con Kaiser, arrojó trece factores que justifican en total 55 items con saturaciones mayores de .30. Su comunalidad podemos considerarla aceptable porque en todos los casos ha sido superior a 0.50 . En conjunto explican un $63 \%$ de la varianza. Dado que el número de factores parecía excesivo, y dada la amplitud de nuestra muestra, decidimos efectuar un análisis factorial de segundo orden. Puede consultarse la matriz factorial rotada en la Tabla 1. El nombre de los factores y la varianza explicada por cada uno de ellos se muestran en la Tabla 2. 
Tabla 1. Matriz factorial rotada del

Cuestionario sobre Valores Sociopersonales para la Convivencia

$\mathrm{N}^{\circ}$ FACTOR 1. Autoexigencia y valía Saturación

Pienso que las personas que tienen un comportamiento estable son mejor

18 aceptadas por los demás.

51 Me siento muy feliz cuando consigo lo que me he propuesto.

8 Considero que ser legal con los demás me hace ser mejor persona.

Me siento muy orgulloso de mí cuando consigo mis metas porque me es-

50 fuerzo.

6 Tengo muy claro que es bueno ser agradecido por los favores que te hacen.

Me produce satisfacción cuando observo que acierto en las cosas que hago,

40 reflexionando cómo las he logrado y reconociéndome mérito por ello.

17 Creo que ser educado es una buena cualidad en las personas.

53 Me siento valioso cuando me piden ayuda los demás.

\section{FACTOR 2. Valor y equilibrio}

38 Me emociona ver que los demás me admiran porque hago cosas atrevidas.

Pienso que podría hacer cosas buenas por los demás que la mayoría de las

7 personas no se atreven.

49 Me agradan las personas que siempre sé como se van a comportar.

\section{FACTOR 3. Respeto y protección}

Me siento desdichado cuando alguien más poderoso que yo arregla las co-

46 sas por la fuerza y no con palabras.

41 Me siento agraciado cuando me valoran positivamente otras personas.

$44 \mathrm{Me}$ entristezco cuando observo que hacen sufrir o padecer daño a otros.

Me altera negativamente mi estado de ánimo observar que no respetan a

57 alguien.

61 Me molesta que se metan con otros que sean inferiores.

\section{FACTOR 4. Bondad y generosidad}

42 Me siento satisfecho cuando tengo que dar cosas personales a los demás.

73 Soy una persona que da cosas personales a otros.

11 Reconozco que me gusta darle cosas personales mías a los demás.

\section{FACTOR 5. Honradez y lucha}

70 Soy una persona que frecuentemente actúa de forma legal con los demás.

85 Soy una persona que lucha contra las injusticias.

92 Soy una persona que procura respetar a los que son más débiles que yo. 
Tengo la costumbre de dedicarle el tiempo y el esfuerzo que haga falta para

81 conseguir lo que me propongo.

\section{FACTOR 6. Amistad, aprecio y amabilidad}

65 Si me veo en apuros, sé que tengo alguien en quien confiar.

34 Me siento a gusto porque tengo amigos en quienes confiar. 72

3 Considero que tengo bastantes amigos.

66 Acostumbro a expresar el valor y admiración que siento por otros. 36

63 Soy una persona que se porta de forma amable con los demás.

\section{FACTOR 7. Prevención de injusticias}

Comprendo que antes de hacer cosas arriesgadas debo pensar en las conse-

12 cuencias que me pueden acarrear. $\quad .74$

13 Pienso que está mal hacerle sufrir o causarle daño a los demás.

23 Estoy convencido que cuando actúo con justicia evito problemas. 38

Tengo la costumbre de pensar las consecuencias de lo que voy a hacer antes

74 de hacerlo.

\section{FACTOR 8. Valor indisciplinado}

Me siento muy enfadado cuando tengo que cumplir las normas establecidas

55 por el Instituto.

69 Soy una persona que hago cosas atrevidas.

\section{FACTOR 9. Protección y equilibrio}

Pienso que los más débiles o distintos por algo (sexo, raza, país de proce-

30 dencia, etc) tienen mayor tendencia a ser perjudicados por otras personas.

Sé gobernar mi propia conducta evitando pasar sin motivos desde una

80 alegría exagerada o una pena muy grande.

\section{FACTOR 10. Altruismo}

10 Pienso que soy una persona bien valorada.

72 Soy una persona a la que reconocen de forma frecuente ser buena persona.

22 Considero que soy una persona que presta ayuda a los demás.

Encuentro emociones agradables cuando, sin esperar nada a cambio, ayudo

33 a los demás.

\section{FACTOR 11. Esfuerzo y orden}

Considero que para mí tiene mucha importancia aprender cosas en el Insti-

5 tuto.

43 Me siento más seguro de mí mismo cuando me paro a pensar en $\quad .35$

las consecuencias de hacer algo arriesgado.

25 Pienso que si todos somos ordenados actuaremos mejor en la vida. 35

64 Soy una persona que me gusta hacer favores a los demás, aunque no me los $\quad-.34$ 
devuelvan.

19 Estoy seguro que esforzándome puedo conseguir lo que yo quiera.

Soy una persona que se esfuerza y presta interés por aprender los conteni-

67 dos de las asignaturas.

Procuro colaborar para que mis materiales y los del instituto estén ordena-

87 dos.

\section{FACTOR 12. Éxito}

Pienso que las personas que tienen éxito en la vida son mejor aceptadas por

20 las demás.

82 Para mí es muy importante obtener éxito.

\section{FACTOR 13. Hospitalidad y reflexión}

21 Pienso que cuando vienen personas de fuera debemos darles buena acogida.

83 Cuando llegan forasteros siempre procuro atenderlos bien.

52 Me siento satisfecho cuando llegan personas forasteras y las tratamos bien.

36 Disfruto aprendiendo los contenidos de las asignaturas y de la tutoría.

Reconozco que las normas y órdenes que nos dan los profesores y los adul-

24 tos son para nuestro bien.

Sé que debo observar mi propia conducta, reflexionar sobre ella y conce-

9 derme cosas agradables cuando actúo de forma apropiada.

En el análisis factorial de segundo orden obtuvimos tres dimensiones. La primera de ellas explica el 43,6\% de la varianza y la hemos denominado "valores sociales y personales" ya que engloba a diez factores de primer orden que presentan estos contenidos, concretamente los factores $1,3,4,5,6,7,9,10,11,13$. La segunda de las dimensiones explica el 11\% de la varianza, la hemos denominado "valor autorregulado" porque engloba dos factores de primer orden ( 2 y 8), que expresan valores relacionados con la autorregulación personal. La tercera dimensión explica el 8\% de la varianza, la hemos denominado "éxito" porque contiene el factor (12) con este mismo contenido semántico. En la tabla 2 puede verse la contribución a la varianza junto con la fiabilidad, de cada factor y de las tres dimensiones. En la Figura 1 puede verse la representación de la estructura factorial de primer y segundo orden del cuestionario. 


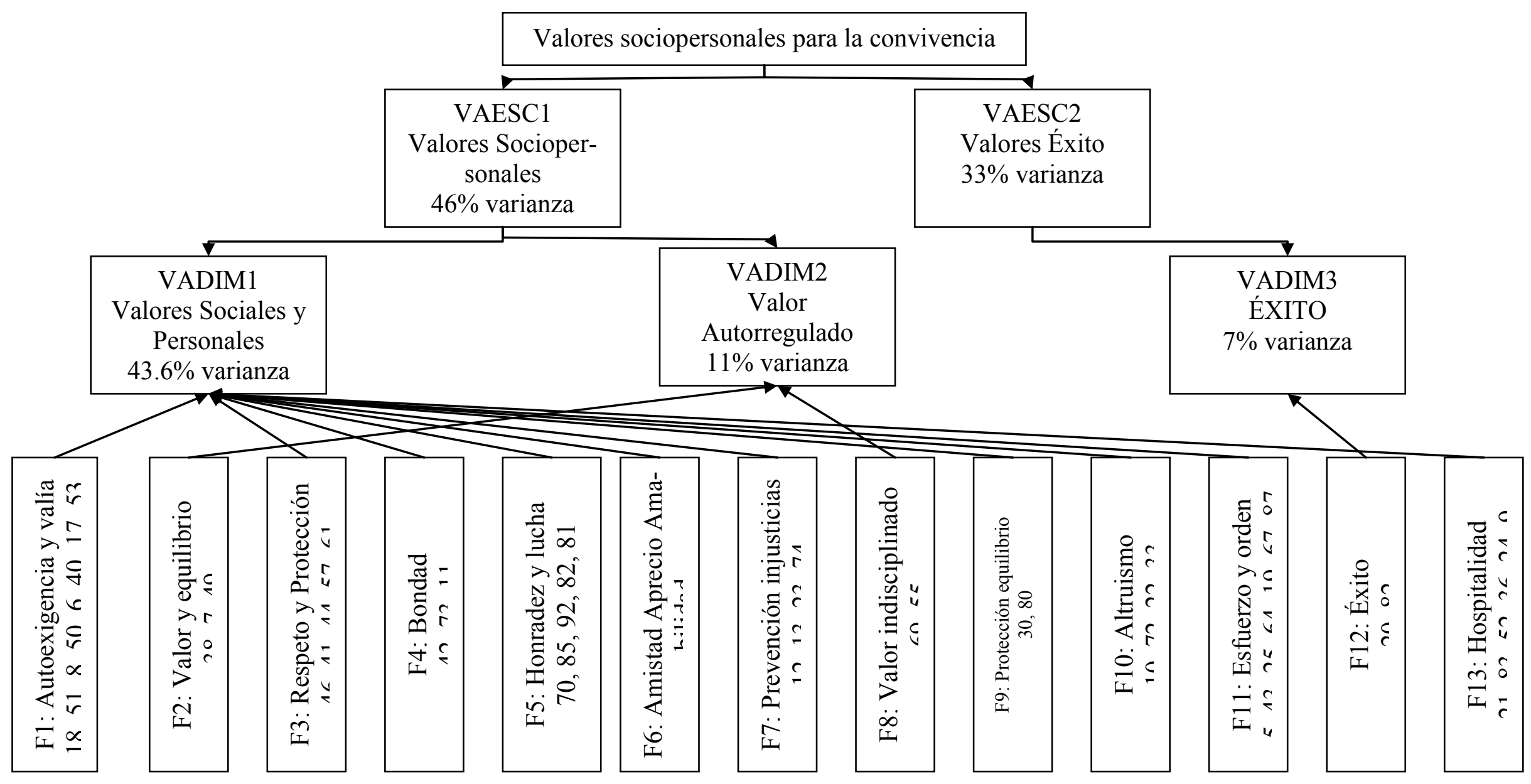




\section{Fiabilidad}

Empleando el coeficiente Alpha de Cronbach como medida de la consistencia interna del cuestionario y de cada uno de los factores que lo componen, obtuvimos un coeficiente global de $.97 \mathrm{y}$, por el método de las dos mitades en la primera de las partes un coeficiente Alpha de .93 y, en la segunda de las partes, un coeficiente de .96. Puede verse en la Tabla 2 el coeficiente Alpha de cada factor de primer orden y de las tres dimensiones del análisis de segundo orden.

Adoptando el criterio de que el coeficiente seleccionado sea superior a .80 (Gingres, 1990), todos los coeficientes obtenidos en las dimensiones son aceptables, al igual que en los factores 1, 11 y 13. En cambio, en el resto de factores los coeficientes de fiabilidad alpha oscilan entre .76 y .39, en función del número de items que contienen (ver Tabla 2).

Tabla 2. Varianza explicada en el análisis factorial, y coeficientes de fiabilidad para la escala total, factores y dimensiones del Cuestionario de Problemas de Convivencia Escolar.

\begin{tabular}{llll}
\hline & $\begin{array}{c}\text { Varianza } \\
\text { explicada }\end{array}$ & & $\begin{array}{c}\text { Coeficiente } \\
\text { Alpha }\end{array}$ \\
\cline { 2 - 3 } Escala total & & & $\alpha=.97$ \\
Dimensión 1: Valores Sociales y Personales & $43,6 \%$ & $\alpha=.93$ \\
Dimensión 2: Valor Autorregulado & $11 \%$ & $\alpha=.71$ \\
Dimensión 3: Valor del Éxito & $7 \%$ & $\alpha=.65$ \\
Factor 1: Autoexigencia y valía & $37 \%$ & $\alpha=.87$ \\
Factor 2: Valor y Equilibrio & $4 \%$ & $\alpha=.39$ \\
Factor 3: Respeto y Protección & $3,2 \%$ & $\alpha=.67$ \\
Factor 4: Bondad & $2,7 \%$ & $\alpha=.76$ \\
Factor 5: Honradez y Lucha & $2,3 \%$ & $\alpha=.76$
\end{tabular}


Tabla 2. Varianza explicada en el análisis factorial, y coeficientes de fiabilidad para la escala total, factores y dimensiones del Cuestionario de Problemas de Convivencia Escolar.

\begin{tabular}{lccc} 
& $\begin{array}{c}\text { Varianza } \\
\text { explicada }\end{array}$ & & $\begin{array}{c}\text { Coeficiente } \\
\text { Alpha }\end{array}$ \\
\cline { 2 - 3 } Factor 6: Amistad, Aprecio y Amabilidad & $2,1 \%$ & & $\alpha=.69$ \\
Factor 7: Prevención de injusticias & $1,9 \%$ & $\alpha=.65$ \\
Factor 8: Valor indisciplinado & $1,8 \%$ & $\alpha=.48$ \\
Factor 9: Protección y equilibrio & $1,7 \%$ & $\alpha=.31$ \\
Factor 10: Altruismo & $1,6 \%$ & $\alpha=.64$ \\
Factor 11: Esfuerzo y Orden & $1,5 \%$ & $\alpha=.87$ \\
Factor 12: Éxito & $1,4 \%$ & $\alpha=.65$ \\
Factor 13: Hospitalidad y reflexión & $1,3 \%$ & $\alpha=.85$
\end{tabular}

\section{Discusión}

La estructura factorial empírica de este cuestionario modifica la que presentaba la versión racional del instrumento, mostrando otra disposición de las dimensiones racionales originales. Esto nos sugiere que el alumnado, en las edades de la adolescencia media, no discrimina entre las dimensiones cognitiva, afectiva-emotiva y conductual, siendo para ellos todo un mismo fenómeno. La primera dimensión empírica tiene un poder explicativo de la varianza muy alto y parece evaluar de manera general valores personales y sociales. De manera global, este alto porcentaje de explicación de la varianza da idea de la tendencia a conformar un único factor o macrovalor sociopersonal.

La dimensión segunda explica un $11 \%$ de la varianza. Sin duda es justificable que los valores de «equilibrio»y, en términos más amplios, «indisciplina» estén en una misma categoría. Además, los valores de indisciplina suponen una grave inadaptación social, que predicen conductas variadas de riesgo futuro de tipo delictivo, adicción a drogas, fracaso y absentismo escolar. La literatura al efecto muestra una estrecha asociación entre conductas de indisciplina, presentar comportamiento antisocial, y comportamientos delictivos de diversos tipos (Farrington y West, 1990, Olweus, 1993). No parece extraño, por tanto, que se asocien 
de forma negativa los comportamientos indisciplinados y los de equilibrio y valía personal, ya que, aunque no tienen la gravedad de otros valores más antisociales, los alumnos, en cuanto a su madurez, son conscientes de que valores de indisciplina retrasan o impiden el proceso de enseñanza y aprendizaje, puesto que no permiten afianzar los aprendizajes (Fernández, 1999, 2001). Este tipo de comportamientos constituye una fuente relevante de problemas para los profesores, ya que aunque sean comportamientos de menor importancia, son muy frecuentes e inapropiados escolar y socialmente.

La tercera dimensión parece especificar la consideración de que las personas que triunfan y que tienen éxito son mejor valoradas por los demás. En este sentido, es muy evidente que el éxito en las instituciones educativas conforma buena parte del autoconcepto y de la autoestima de los adolescentes, transmitiendo una especie de mensaje simbólico de que cuando las calificaciones escolares son aptas, los jóvenes se ven mejor considerados en la institución escolar, familiar y social (Ortega y del Rey, 2003), interpretando el propio afectado de que se están cumpliendo las expectativas sociales que en él se han depositado.

Podemos afirmar, por tanto, que el cuestionario mide valores importantes en contextos escolares según el punto de vista del propio alumno (ver formato racional completo en el Anexo I). Su utilidad reside en la exploración sistemática de distintos tipos de valores importantes que pueden aparecer en el ámbito de la convivencia en los centros escolares, agrupándolos en tres núcleos de distinto significado: a) valores de tipo personal y social; b) valores relacionados con la valía y la autorregulación conductual, en la medida en que contribuyen a mejorar el clima de aprendizaje y trabajo en el centro escolar; y, c) valores relacionados con éxito. Cuando estos valores influyen en sentido negativo son un núcleo de contenido importante para la predicción de fracaso escolar, abandono y salida del sistema educativo.

Como instrumento de evaluación puede facilitar la identificación de alumnos con valores contrarios a las normas de convivencia, facilitando la intervención en el aula y, simultáneamente, orientando a los profesionales que trabajan con estos alumnos para elaborar programas de intervención más eficaces respecto a otras variables sociodemográficas y académicas de los alumnos. No obstante, será preciso continuar estudiando la estructura factorial confirmatoria de este instrumento, ya que habrá que descartar si su estructura factorial tiende a factor único, junto a la implementación de algunos de los factores con objeto de mejorar sus coeficientes de fiabilidad. 
Estudio 2. Perfil de los valores sociopersonales para la convivencia según diferentes variables de los alumnos

\section{Método}

\section{Procedimiento}

Utilizamos el instrumento de recogida de información personal, familiar y contextual elaborado y validado por nosotros, denominado Hoja de recogida de variables sociodemográficas (Peralta, Sánchez, De la Fuente y Trianes, 2003). El alumnado cumplimentó dicha hoja en una sesión previa e independiente a la del Cuestionario de Valores Sociopersonles. En el caso de cuestiones incompletas o dudosas se confirmó en entrevistas posteriores con los interesados la información pertinente.

\section{Análisis estadísticos}

Realizamos análisis multivariados tomando como variables independientes el género, con dos niveles (varón, mujer); grupos de edad, con dos niveles (12-14 años y 15-17 años); repetición en la ESO, con dos niveles (sí repiten y no repiten) y estructura familiar con cuatro niveles (familia extensa: vive con abuelos, tíos u otros familiares; 2 . vive sólo con el padre; 3. vive solo con la madre y 4 . familias completas). Como variables dependientes se han tomado las distintas puntuaciones del cuestionario sobre valores sociopersonales para la convivencia.

\section{Resultados}

En cuanto al género, Los resultados estadísticos nos permiten afirmar que, en líneas generales, las alumnas obtienen mayores puntuaciones en los valores que los alumnos, para cada una de las puntuaciones del Cuestionario, excepto en la dimensión tercera y el factor cuarto (valor del éxito), en los que se invierten los resultados (véase la Tabla 3).

\begin{tabular}{c}
\multicolumn{7}{c}{ Tabla 3. Relaciones de interdependencia entre el Género y las puntuaciones del Cuestionario de } \\
Valores Sociopersonales para la Convivencia
\end{tabular}




\begin{tabular}{llllll}
\hline F1 & $3,99(0,79)$ & $4,14(0,73)$ & $\mathrm{F}(1,855)=8,040^{* *}$ & $1<2$ \\
F2 & $3,47(0,75)$ & $3,53(0,70)$ & $\mathrm{F}(1,855)=1,200$ n.s. & $1<2$ & \\
F3 & $3,35(0,80)$ & $3,65(0,79)$ & $\mathrm{F}(1,855)=31,566^{* * * *}$ & $1<2$ & \\
F4 & $3,53(1,07)$ & $3,38(1,07)$ & $\mathrm{F}(1,855)=3,923^{*}$ & $1>2$ & \\
F5 & $3,68(0,73)$ & $3,82(0,67)$ & $\mathrm{F}(1,855)=8,451^{* *}$ & $1<2$ & \\
F6 & $3,76(0,67)$ & $3,95(0,64)$ & $\mathrm{F}(1,855)=18,730^{* * * *}$ & $1<2$ & $\mathrm{~F}(13,843)=7840,906^{* * * *}$ \\
F7 & $3,72(0,77)$ & $3,89(0,76)$ & $\mathrm{F}(1,855)=10,751^{* * *}$ & $1<2$ & \\
F8 & $2,76(1,03)$ & $2,99(0,98)$ & $\mathrm{F}(1,855)=11,518^{* * *}$ & $1<2$ & \\
F9 & $3,59(0,91)$ & $3,57(0,86)$ & $\mathrm{F}(1,855)=, 065$ n.s. & n.s. & \\
F10 & $3,44(0,71)$ & $3,58(0,71)$ & $\mathrm{F}(1,855)=7,873^{* *}$ & $1<2$ & \\
F11 & $3,57(0,94)$ & $3,77(0,87)$ & $\mathrm{F}(1,855)=9,957^{* * *}$ & $1<2$ & \\
F12 & $3,79(1,18)$ & $3,55(1,29)$ & $\mathrm{F}(1,855)=7,712^{* *}$ & $1>2$ & \\
F13 & $3,24(0,99)$ & $3,46(0,87)$ & $\mathrm{F}(1,855)=12,429 * * * *$ & $1<2$ & \\
\hline$* \mathrm{p}<.05 ; * * \mathrm{p}<.01 ; * * * \mathrm{p}<.001 ; * * * * \mathrm{p}<.0001$ & &
\end{tabular}

Para los grupos de edad, el grupo de adolescentes de menor edad (12-14 años) obtienen mayores puntuaciones que los de mayor edad (15-17 años). Los resultados se presentan en la Tabla 4.

Tabla 4. Relaciones de interdependencia entre Grupos de edad y las puntuaciones del Cuestionario de Valores Sociopersonales para la Convivencia

\begin{tabular}{|c|c|c|c|c|c|}
\hline & 12-14 años & 15-17 años & F parcial (traza de & Post hoc & \multirow[t]{3}{*}{ F total (Traza de Pillai) } \\
\hline & $\operatorname{Media}(S d)$ & $\operatorname{Media}(S d)$ & \multirow{2}{*}{\multicolumn{2}{|c|}{ Pillai) }} & \\
\hline & $\mathrm{N}=439$ & $\mathrm{~N}=418$ & & & \\
\hline D1 & $3,76(0,51)$ & $3,54(0,48)$ & $\mathrm{F}(1,855)=44,021 * * * *$ & $1>2$ & \\
\hline D2 & $3,26(0,59)$ & $3,11(0,48)$ & $\mathrm{F}(1,855)=17,388 * * * *$ & $1>2$ & $\mathrm{~F}(3,853)=15,653 * * * *$ \\
\hline D3 & $3,67(1,31)$ & $3,68(1,17)$ & $\mathrm{F}(1,855)=, 028$ n.s. & n.s. & \\
\hline F1 & $4,16(0,72)$ & $3,97(0,79)$ & $\mathrm{F}(1,855)=12,389 * * * *$ & $1>2$ & \\
\hline $\mathrm{F} 2$ & $3,52(0,79)$ & $3,48(0,64)$ & $\mathrm{F}(1,855)=, 493$ n.s. & n.s. & \\
\hline F3 & $3,65(0,84)$ & $3,34(0,75)$ & $\mathrm{F}(1,855)=31,542 * * * *$ & $1>2$ & \\
\hline F4 & $3,43(1,05)$ & $3,49(1,08)$ & $\mathrm{F}(1,855)=, 688$ n.s. & n.s. & \\
\hline F5 & $3,87(0,71)$ & $3,62(0,67)$ & $\mathrm{F}(1,855)=28,185 * * * *$ & $1>2$ & \\
\hline F6 & $3,92(0,68)$ & $3,79(0,63)$ & $\mathrm{F}(1,855)=8,364 * *$ & $1>2$ & \\
\hline F7 & $3,96(0,79)$ & $3,64(0,72)$ & $\mathrm{F}(1,855)=38,571 * * * *$ & $1>2$ & $\mathrm{~F}(13,843)=7,010 * * * *$ \\
\hline F8 & $3,00(1,07)$ & $2,73(0,93)$ & $\mathrm{F}(1,855)=15,837 * * * *$ & $1>2$ & \\
\hline F9 & $3,72(0,86)$ & $3,44(0,90)$ & $\mathrm{F}(1,855)=20,846^{* * * *}$ & $1>2$ & \\
\hline F10 & $3,57(0,73)$ & $3,45(0,68)$ & $\mathrm{F}(1,855)=6,227 * *$ & $1>2$ & \\
\hline F11 & $3,87(0,84)$ & $3,46(0,94)$ & $\mathrm{F}(1,855)=45,273 * * * *$ & $1>2$ & \\
\hline F12 & $3,67(1,31)$ & $3,68(1,17)$ & $\mathrm{F}(1,855)=, 028$ n.s. & n.s. & \\
\hline F13 & $3,51(0,92)$ & $3,19(0,93)$ & $\mathrm{F}(1,855)=25,549 * * * *$ & $1>2$ & \\
\hline
\end{tabular}


Según la estructura familiar, en general, los que viven con familias extensas (abuelos, tíos, etc) tienen puntuaciones menores que los que viven sólo con los padres o con familias compactas (factores 6 y 11). Del mismo modo, aquellos alumnos que viven sólo con el padre tienen mayores puntuaciones que los que viven solos con las madres (dimensión 1). A su vez, los que viven sólo con las madres tienen menores puntuaciones que los que viven en familias compactas con todos sus miembros (dimensión 1 y factores $1,5,7,8,9,10,11$ y 13), haciendo la salvedad del factor cuarto en el que los que viven sólo con las madres tienen mayores puntuaciones que los que viven con familias compactas. No obstante, el efecto más claro es que los alumnos que viven en familias completas (número 4) tienen, en general, más valores sociopersonales para la convivencia. Para el "valor del éxito" (dimensión 3 y factor 12) no aparece un efecto significativo de la variable tipo de familia (ver Tabla 5).

Tabla 5. Relaciones de interdependencia entre Estructura familiar y las puntuaciones del Cuestionario de Valores Sociopersonales para la Convivencia

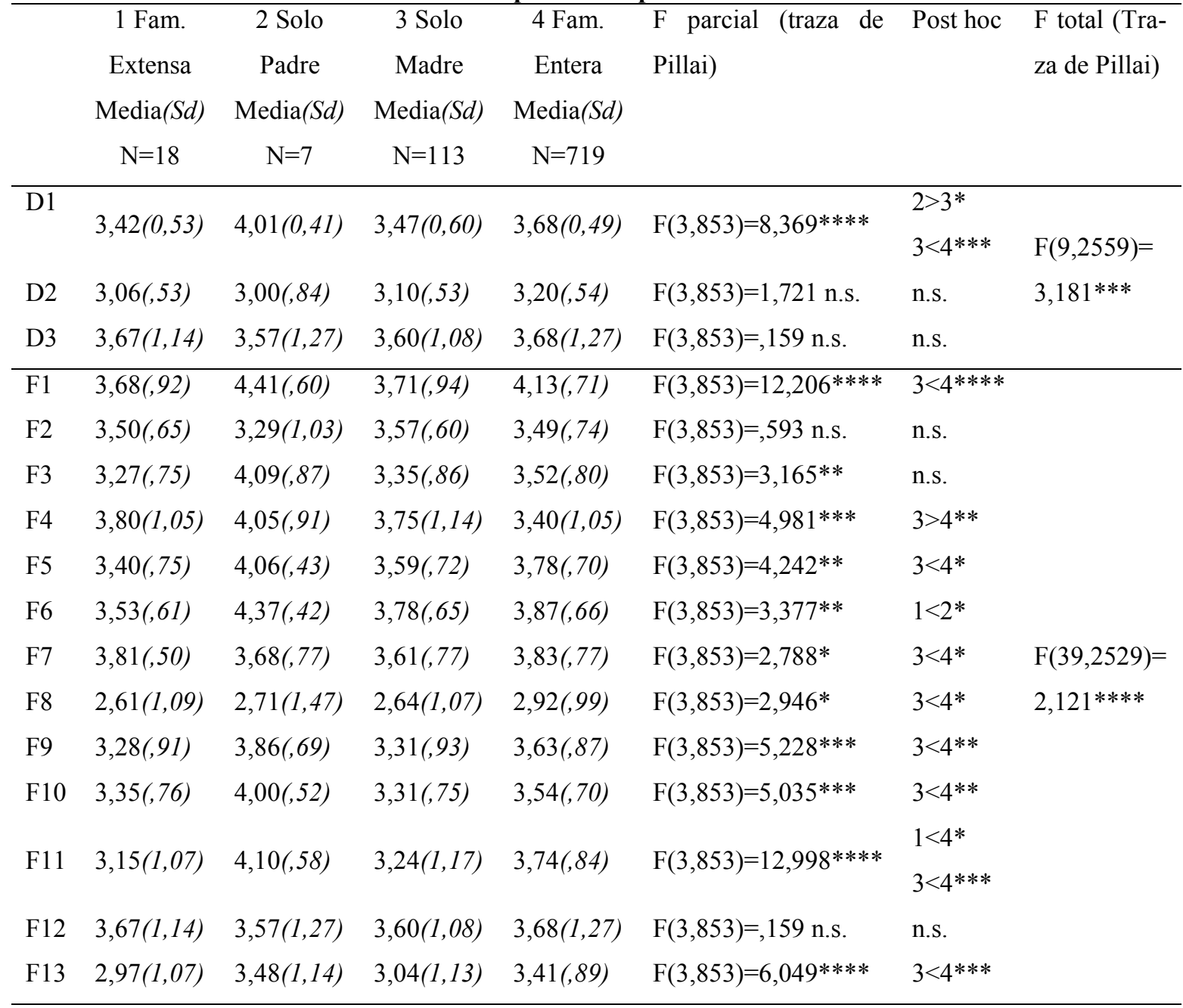

$* \mathrm{p}<.05 ; * * \mathrm{p}<.01 ; * * * \mathrm{p}<.001 ; * * * * \mathrm{p}<.0001$ 
Los resultados concernientes a los valores para la convivencia tomando como variable independiente la Repetición en la E.S.O. arrojan resultados estadísticamente significativos apuntando que, en general, los repetidores obtienen menores valores que los no repetidores. Los resultados se presentan en la Tabla 6.

Tabla 6. Relaciones de interdependencia entre Repetición en la ESO y las puntuaciones del Cuestionario de Valores Sociopersonales para la Convivencia

\begin{tabular}{|c|c|c|c|c|c|}
\hline & Sí Rp ESO & No Rp ESO & F parcial (traza de & Post hoc & \multirow[t]{3}{*}{ F total (Traza de Pillai) } \\
\hline & $\operatorname{Media}(S d)$ & $\operatorname{Media}(S d)$ & \multirow{2}{*}{\multicolumn{2}{|c|}{ Pillai) }} & \\
\hline & $\mathrm{N}=241$ & $\mathrm{~N}=616$ & & & \\
\hline D1 & $3,43(0,50)$ & $3,74(0,49)$ & $\mathrm{F}(1,855)=66,771 * * * *$ & $1<2$ & \\
\hline D2 & $3,06(0,46)$ & $3,24(0,57)$ & $\mathrm{F}(1,855)=19,011 * * * *$ & $1<2$ & $\mathrm{~F}(3,853)=16961,224 * * * *$ \\
\hline D3 & $3,70(1,12)$ & $3,66(1,29)$ & $\mathrm{F}(1,855)=, 135$ n.s. & n.s. & \\
\hline F1 & $3,82(0,88)$ & $4,16(0,69)$ & $\mathrm{F}(1,855)=37,394 * * * *$ & $1<2$ & \\
\hline $\mathrm{F} 2$ & $3,55(0,64)$ & $3,48(0,75)$ & $\mathrm{F}(1,855)=1,588$ n.s. & & \\
\hline F3 & $3,26(0,75)$ & $3,59(0,82)$ & $\mathrm{F}(1,855)=28,259 * * * *$ & $1<2$ & \\
\hline $\mathrm{F} 4$ & $3,53(1,12)$ & $3,43(1,05)$ & $F(1,855)=1,545$ n.s. & & \\
\hline F5 & $3,50(0,66)$ & $3,84(0,70)$ & $\mathrm{F}(1,855)=43,216^{* * * *}$ & $1<2$ & \\
\hline F6 & $3,74(0,62)$ & $3,90(0,68)$ & $\mathrm{F}(1,855)=9,380 * * *$ & $1<2$ & \\
\hline F7 & $3,57(0,75)$ & $3,89(0,76)$ & $\mathrm{F}(1,855)=32,132 * * * *$ & $1<2$ & $\mathrm{~F}(13,843)=8,535 * * * *$ \\
\hline F8 & $2,56(0,92)$ & $2,99(1,02)$ & $\mathrm{F}(1,855)=31,868 * * * *$ & $1<2$ & \\
\hline F9 & $3,34(0,95)$ & $3,68(0,85)$ & $\mathrm{F}(1,855)=25,502 * * * *$ & $1<2$ & \\
\hline F10 & $3,35(0,71)$ & $3,57(0,70)$ & $\mathrm{F}(1,855)=17,461 * * * *$ & $1<2$ & \\
\hline F11 & $3,23(1,02)$ & $3,84(0,80)$ & $\mathrm{F}(1,855)=86,959 * * * *$ & $1<2$ & \\
\hline F12 & $3,70(1,12)$ & $3,66(1,29)$ & $\mathrm{F}(1,855)=, 135$ n.s. & & \\
\hline F13 & $3,00(0,99)$ & $3,48(0,89)$ & $\mathrm{F}(1,855)=47,857 * * * *$ & $1<2$ & \\
\hline
\end{tabular}

Estudio 3. Interdependencia entre los valores sociopersonales para la convivencia y la conducta antisocial

\section{Método}

Instrumentos

El instrumento utilizado en este trabajo es el The School Social Behavior Scales, SSBS (Merrell, 1993). Para nuestro estudio efectuamos la traducción de la Escala B, referida a la Conducta Antisocial. El instrumento traducido que han contestado los profesores se denomina Cuestionario de Conducta Antisocial. Esta versión consta de 33 items y cinco niveles 
de respuesta (En absoluto, Un poco, Algo, Bastante, Mucho). Algunos ejemplos de items son: "molesta a otros estudiantes con sus problemas"; "coge cosas de los demás"; "desafía al personal de la escuela"; "destruye o daña las cosas de la escuela"; "tiene un temperamento con arranques o rabietas"; "utiliza un lenguaje vulgar y obsceno", etc.

Los profesores tutores de cada grupo-clase realizaron la evaluación, alumno por alumno. Su puntuación total evalúa la conducta antisocial que muestra un estudiante, desde el punto de vista del profesor. Para la Escala de Conducta Antisocial (Merrell, 1993), el equipo de investigación se reunía con cada tutor/a del grupo clase y juntos procedían a la evaluación de cada alumno.

\section{Análisis estadísticos}

En primer lugar, dividimos al conjunto de los sujetos en tres grupos (altos, medios y bajos) en función de la puntuación obtenida en la conducta antisocial evaluada por el profesorado en la prueba Escala B (Conducta Antisocial) de Merrell (1993). El criterio escogido ha sido en este caso el análisis de cluster en tres grupos, ya que la varianza no es homogénea. En segundo lugar, se efectuaron dos análisis de varianza multivariados, utilizando como variable independiente la puntuación en conducta antisocial y como variables dependientes las tres dimensiones y los trece factores del cuestionario sobre valores sociopersonales de convivencia escolar.

\section{Resultados}

Como se esperaba, los tres grupos se diferencian significativamente en cada uno de las dimensiones y factores del Cuestionario sobre Valores Sociopersonales para la Convivencia Escolar. A mayor conducta antisocial poseída, se presenta una puntuación más baja en las dimensiones y factores en los valores sociopersonales (véanse los resultados en la Tabla 7).

Tabla 7. Valores de los análisis multivariados en relación con las dimensiones y factores del Cuestionario sobre Valores Sociopersonales, según los grupos de baja, media y alta Conducta Antisocial.

\begin{tabular}{|c|c|c|c|c|c|c|}
\hline & $\begin{array}{l}\text { Baja } \\
(n=557)\end{array}$ & $\begin{array}{l}\text { Media } \\
(n=219)\end{array}$ & $\begin{array}{l}\text { Alta } \\
(n=81)\end{array}$ & F parcial (Pillai) & Post hoc & $\begin{array}{l}\text { F Total } \mathrm{p}< \\
\text { (traza Pillai) }\end{array}$ \\
\hline Dimensiones y Factores & $\mathrm{M}(d t)$ & $\mathrm{M}(d t)$ & $\mathrm{M}(d t)$ & & & \\
\hline \multirow{2}{*}{ D1: Valores Personales y Sociales } & \multirow{2}{*}{$3,76(, 42)$} & \multirow{2}{*}{$3,53(, 57)$} & \multirow{2}{*}{$3,25(, 61)$} & $\mathrm{F}(2,854)=$ & $1>2>3^{* \star *}$ & \multirow[b]{2}{*}{$\mathrm{F}(6,1706)=$} \\
\hline & & & & $46,979 * * * *$ & $2>3^{* * *}$ & \\
\hline D2: Valores de Autorregulación & $3,28(, 53)$ & $3,05(, 51)$ & $2,91(, 52)$ & $\begin{array}{l}\mathrm{F}(2,854)= \\
26,597 * * * *\end{array}$ & $1>2>3^{* * * *}$ & $17,746^{* * * *}$ \\
\hline
\end{tabular}


Tabla 7. Valores de los análisis multivariados en relación con las dimensiones y factores del Cuestionario sobre Valores Sociopersonales, según los grupos de baja, media y alta Conducta Antisocial.

\begin{tabular}{|c|c|c|c|c|c|c|}
\hline & $\begin{array}{l}\text { Baja } \\
(\mathrm{n}=557)\end{array}$ & $\begin{array}{l}\text { Media } \\
(n=219)\end{array}$ & $\begin{array}{l}\text { Alta } \\
(n=81)\end{array}$ & F parcial (Pillai) & Post hoc & $\begin{array}{l}\text { F Total } \mathrm{p}< \\
\text { (traza Pillai) }\end{array}$ \\
\hline Dimensiones y Factores & $\mathrm{M}(d t)$ & $\mathrm{M}(d t)$ & $\mathrm{M}(d t)$ & & & \\
\hline D3: Búsqueda de éxito & $3,66(1,24)$ & $3,64(1,28)$ & $3,82(1,24)$ & $\begin{array}{l}\mathrm{F}(2,854)= \\
, 711 \text { n.s. }\end{array}$ & n.s. & \\
\hline F1: Autoexigencia y Valía & $4,24(, 57)$ & $3,89(, 89)$ & $3,38(1,00)$ & $\begin{array}{l}\mathrm{F}(2,854)= \\
60,587 * * * *\end{array}$ & $\begin{array}{l}1>2>3^{\star * * *} \\
2>3^{\star * * *}\end{array}$ & \\
\hline F2: Equilibrio & $3,46(, 72)$ & $3,58(, 71)$ & $3,56(, 76)$ & $\begin{array}{l}\mathrm{F}(2,854)= \\
2,446 \text { n.s. }\end{array}$ & n.s. & \\
\hline F3: Respeto y Protección & $3,62(, 75)$ & $3,36(, 87)$ & $3,01(, 83)$ & $\begin{array}{l}\mathrm{F}(2,854)= \\
25,944 * * *\end{array}$ & $\begin{array}{l}1>2^{\star *} \\
1>3^{\star * * *}\end{array}$ & \\
\hline F4: Bondad & $3,28(1,01)$ & $3,71(1,10)$ & $3,95(, 09)$ & $\begin{array}{l}\mathrm{F}(2,854)= \\
22,814 * * * *\end{array}$ & $\begin{array}{l}1<2^{\star \star * *} \\
1<3^{\star * * *}\end{array}$ & \\
\hline F5: Honradez y lucha & $3,85(, 66)$ & $3,61(, 71)$ & $3,37(, 76)$ & $\begin{array}{l}\mathrm{F}(2,854)= \\
22,800 * * * *\end{array}$ & $\begin{array}{l}1>2>3^{* \star * *} \\
2>3^{* \star * *}\end{array}$ & \\
\hline F6: Amistad, Precio y Amabilidad & $3,92(, 65)$ & $3,82(, 64)$ & $3,47(, 65)$ & $\begin{array}{l}\mathrm{F}(2,854)= \\
16,964 * * * *\end{array}$ & $\begin{array}{l}1>3^{* * * *} \\
2>3^{* \star * *}\end{array}$ & \\
\hline F7: Prevención de injusticias & $3,91(, 73)$ & $3,60(, 84)$ & $3,56(, 68)$ & $\begin{array}{l}\mathrm{F}(2,854)= \\
17,400 * * * *\end{array}$ & $\begin{array}{l}1>2^{* \star * *} \\
1>3^{* * *}\end{array}$ & $\begin{array}{l}\mathrm{F}(26,1686)= \\
8,282 * * *\end{array}$ \\
\hline F8: Valor indisciplinado & $3,09(, 94)$ & $2,53(, 99)$ & $2,25(1,00)$ & $\begin{array}{l}\mathrm{F}(2,854)= \\
45,827 * * * *\end{array}$ & $\begin{array}{l}1>2^{* * * *} \\
1>3^{* * * *}\end{array}$ & \\
\hline F9: Protección y equilibrio & $3,67(, 87)$ & $3,48(, 90)$ & $3,25(, 88)$ & $\begin{array}{l}\mathrm{F}(2,854)= \\
9,937 * * * *\end{array}$ & $\begin{array}{l}1>2^{* *} \\
1>3^{* * * *}\end{array}$ & \\
\hline F10: Altruismo & $3,60(, 65)$ & $3,43(, 76)$ & $3,11(, 71)$ & $\begin{array}{l}\mathrm{F}(2,854)= \\
19,336 * * * *\end{array}$ & $\begin{array}{l}1>2^{* *} \\
1>3^{* * * *}\end{array}$ & \\
\hline F11: Esfuerzo y orden & $3,91(, 64)$ & $3,36(1,05)$ & $2,80(1,27)$ & $\begin{array}{l}\mathrm{F}(2,854)= \\
82,290 * * * *\end{array}$ & $\begin{array}{l}1>2>3^{* * * *} \\
2>3^{* * * *}\end{array}$ & \\
\hline F12: Éxito & $3,66(1,24)$ & $3,64(1,29)$ & $3,83(1,13)$ & $\begin{array}{l}\mathrm{F}(2,854)= \\
, 711 \text { n.s. }\end{array}$ & n.s. & \\
\hline F13: Hospitalidad y reflexión & $3,56(, 79)$ & $3,08(1,02)$ & $3,63(1,13)$ & $\begin{array}{l}\mathrm{F}(2,854)= \\
51,715 * * *\end{array}$ & $\begin{array}{l}1>2^{\star * * *} \\
2<3^{\star * *}\end{array}$ & \\
\hline
\end{tabular}

Las tres dimensiones del cuestionario sobre los valores de convivencia escolar, en general, diferencian significativamente entre los grupos bajos, medios y altos, formados por la puntuación en conducta antisocial. Ésta puede ser definida como la inadaptación y la ausencia de logro de importantes objetivos sociales en contextos específicos. Los jóvenes que manifiestan conductas antisociales utilizan medios inapropiados y obtienen resultados negativos escolares, sociales y, posteriormente, laborales para el desarrollo del alumno (Ford, 1982, Cavell, 1990). Los valores humanos forman un componente importante del ajuste y de la adaptación sociopersonal y escolar (De la Fuente, 1999, 2000). En este estudio, los alumnos que muestran mayor puntuación en conducta antisocial presentan, en general, más baja pun- 
tuación en las dimensiones y factores de los valores sociopersonales para la convivencia escolar, evidenciando una fuerte relación negativa entre ambos instrumentos.

\section{Conclusiones generales}

Podemos concluir afirmando que en el primer estudio, cuyo objetivo era la elaboración de un nuevo instrumento para la evaluación de valores relacionados con la convivencia escolar, los resultados han confirmado unas propiedades psicométricas aceptables para esta versión inicial del instrumento, si nos atenemos a los índices de fiabilidad y validez. En el mismo sentido, estos resultados nos sugieren la necesidad de seguir indagando en la estructura interna, mediante análisis confirmatorios, y en la fiabilidad del cuestionario, a fin de mejorar algunos índices.

Entre todos los resultados obtenidos cabe destacar, desde nuestro punto de vista, el gran peso que tiene el valor "éxito social" que con muy pocos items explica una gran cantidad de varianza como la dimensión segunda de nuestro constructo "Valores sociopersonales". Además, en la dimensión primera, referida a los "Valores sociales y personales", de forma coherente con el resultado anterior, el factor que tiene más peso es el de "autoexigencia y valía personal", referido en gran medida a la consecución de metas personales y sociales. Este resultado no nos debe resultar extraño si tenemos en cuenta la gran presión social de los diferentes contextos educativos a los que están expuestos los alumnos adolescentes para conseguir logros académicos, personales y sociales.

En cuanto al segundo estudio, la relación de interdependencia de los valores con determinadas variables sociodemográficas es importante. Hemos confirmado que, en general, las alumnas obtienen mejores puntuaciones en valores sociales que los alumnos, excepto en lo referido a los valores éxito, en el que los chicos superan a las chicas. Este resultado debería hacernos reflexionar de cara a la intervención educativa, habida cuenta que los alumnos muestran un peor nivel de incorporación en valores sociopersonles, lo que supondría considerarlos como población de mayor riesgo en el proceso educativo.

El resultado anterior, además, se matiza con la edad. Para los grupos de edad, aparece un deterioro de los valores en el grupo de mayor edad. En este caso, alcanzar mayor edad, en contra de lo que sería esperable, lleva consigo una considerable disminución en los valores sociopersonales para la convivencia. Probablemente haya que buscar la causa en la crisis personal y social que la adolescencia lleva consigo. Sin embargo, tampoco debe ser menospre- 
ciada la influencia de los medios sociales y de los valores que el propio entorno ejerce, a través de los múltiples contextos de socialización.

Respecto a la estructura familiar, aparece un resultado reiterativo indicando que los hijos que viven en familias desestructuradas experimentan menor construcción de valores sociopersonles. En cualquier caso, nuestros resultados ahondan en el valor del papel de la familia estructurada como medio de socialización, entendido como construcción de valores sociopersonales, de manera coherente con otras investigaciones previas (Arranz, Yenes, Olabarrieta y Martín, 2001).

La relación de los valores sociopesonales también ha aparecido como interdependiente con el fracaso escolar. Los alumnos repetidores en la E.S.O. también obtienen menores puntuaciones que los no repetidores. Estos resultados apoyan evidencias previas, referentes al peor desarrollo social y comportamientos desadaptativos de los alumnos repetidores (García, De la Fuente, Justicia y cols., 2002)

Finalmente, el tercer estudio, además de confirmar la validez externa del cuestionario que se ha elaborado, apunta de forma muy consistente que, a mayor conducta antisocial, los estudiantes poseen menores valores. Este resultado ha permitido obtener un correlato fiable de los valores sociopersonales, mostrando que el comportamiento antisocial, además de ser un problema de competencias o habilidades sociales, como se aborda desde numerosos modelos psicológicos, ya clásicos, es decir, de competencias procedimentales, es un problema de ausencia de valores sociopersonales para la convivencia (De la Fuente, 1999).

Por tanto, entendemos que nuestros resultados suponen un auténtico espaldarazo a la necesidad de evaluar y educar en valores sociopersonales para la convivencia a los alumnos de la etapa de Educación Secundaria, en la línea de otros trabajos recientes (Iriarte, Alonso y Sobrino, 2006) De ello depende, en buena medida, su futuro y el de todos nosotros, como sociedad más desarrollada y, a la vez, más humana y civilizada. 


\section{Referencias}

Arranz, E., Yenes, F., Olabarrieta, F. y Martín, J.L. (2001). Relaciones entre hermanos y desarrollo psicológico en escolares. Infancia y Aprendizaje, 24(3), 361-377.

Bilsky, W. y Schwartz, S.H. (1994). Values and Personality. European Journal of Personality, $8,163-181$.

Braithwaite, V.A. y Law, H. G. (1985). Structure of human values: testing the adequacy of the Rokeach Value Survey. Journal of Personality abd Social Psychology, 49, 250-263.

Cavell, T.A. (1990). Social adjustment, social performance and social skills: A tri-component modelo of social competence. Journal of Clinical Child Psychology, 19, 111-122.

De la Fuente, J. (1999). Educación para la construcción personal. Un enfoque de autorregulación en la formación de profesores y alumnos. Bilbao. Desclée de Brouwer.

De la Fuente, J. (2000). Taller de mejora personal: aprendemos a regular nuestros pensamientos, sentimientos y acciones. En M. Álvarez y R. Bisquerra, Manual de Orientación y Tutoría (pp. 330/225-330/239). Barcelona: CissPraxis.

De la Fuente, J. (2003). Un enfoque de autorregulación en la Educación de Valores: Teoría y práctica. Conferencia Plenaria en el Congreso Estatal de Educación en Valores. Guadalajara (México), 6 de Noviembre. Manuscrito sin publicar.

Farrington, D. P. (1989). Early predictors of adolescent aggression and adult violence. Violence and Victims, 4, 79-100.

Farrington, D.P. (1993). Understanding and preventing bullying. En M. Tonry (Ed.), Crime and justice: A review of research (vol. 17) (pp. 381-458). Chicago: University of Chicago Press.

Farrington. D.P. y West, D. (1990). The Cambridge study in delinquent development a longterm follow-up of 411 London males. En H.J. Kerner y G.Kaiser (Eds), Criminality: personality, behavior and life history. Berlin: Springer Verlag.

Fernández, I. (1999). Prevención de la violencia y resolución de conflictos. Madrid: Narcea.

Fernández, I. (2001). Guía para la convivencia en el aula. Madrid: Ciss-praxis.

Ford, M.E. (1982). Social cognition and social competence in adolescence. Development Psychology, 18, 323-340.

García, M., De la Fuente, J., Justicia, F. y cols. (2002). Autorregulación del aprendizaje en el aula. Sevilla : Consejería de Educación. Junta de Andalucía. 
Gingres, M. (1990). Elaboration d'une stratégie d'evaluation des besoins d'education à la carrière chez les finissants du secondaire. Tesis Doctoral. Sherbrooke: Université de Sherbrooke.

Iriarte, C., Alonso, N. y Sobrino, A. (2006). Relaciones entre el desarrollo emocional y moral a tener en cuenta en el ámbito educativo: propuesta de un programa de intervención. Revista de Investigación Psicoeducativa, 4(1), 43-64.

Kazdin, A.E. y Buela-Casal, G. (1996). Conducta antisocial. Madrid: Ed. Pirámide.

Levy, S. y Guttman, L. (1974). Values and attitudes of Israeli high school students. Jerusalem: Israel. Institute of Aplied Social Research.

Markus, H. y Kitayama, S. (1991). Culture and the self: Implications for cognition, emotion and motivation. Psychological, 98, 224-253.

Merrell, K.W. (1993). Using behavior rating scales to assess social skills and antisocial behavior in school settings: Development of the school social behavior scales. School Psychology Review, 22 (1), 115-133.

Olweus, D. (1993). Bullying at school: What know and what we can do. Oxford: Blackwell.

Ortega, R. (2000). A Global, Ecological and Cultural Model for Dealing with Problems of Violence in European Compulsory Schools. Vith Meeting of TMR Programme: Nature and Prevention of Bullying and Social Exclusion. Cruz Quebrada-Dafundo. Lisboa.

Ortega, R. y Del Rey, R. (2003). La violencia escolar. Estrategias de prevención. Barcelona: Graó.

Peralta, F.J., Sánchez, M.D., Trianes, M.V. y De la Fuente (2003). Estudio de la validez interna y externa de un cuestionario sobre conductas problemáticas para la convivencia según el profesor. Psicologia, Saúde \& Doenças, 4(1), 83-96.

Schmitt, M.J., Schuwartz, S.H., Steyer, R. y Schmitt, T. (1993). Measurement models for the Schwartz Values Inventory. European Journal of Personality Assessment, 9, 107-121.

Schwartz, S.H. (1994). Are there Universal Aspects in the Structure and Contents of human Values? Journal of Social Issues, 50(4), 19-45

Schwartz, S.H. y Sagiv, L. (1995). Identifying culture-specifics in the content and structure of values. Journal of Cross-Cultural Psycholgy, 26, 92-126.

Smith, P.K., Morita, Y., Junger-Tas, J., Olweus, D., Catalano, R. y Slee, P. (1999). The nature of school bullying. Londres: Routledge.

Tierno, B. (1992). Valores humanos II. Madrid: Taller de Editores S.A.

Tierno, B. (1993 a). Valores humanos I. Madrid: Taller de Editores S.A. 
Tierno, B. (1993 b). Valores humanos III. Madrid: Taller de Editores S.A.

Tierno, B. (1996). Guía para educar en los valores humanos. Madrid: Taller de Editores S.A. Tremblay, R.E., Kurtz, L., Masse, L., Vitaro, F. y Phil, R.O. (1995). A bimodal preventive intervention for disruptive kindergarten boys: It's impact through adolescence. Journal of Consulting and Clinical Psychology, 63(4), 560-568.

Trianes, M.V., Blanca, M.J. Muñoz, A. García, B. Cardelle-Elawar, M. e Infante, L. (2002). Relaciones entre evaluadores de la competencia social en preadolescentes: Profesores, iguales y autoinformes. Anales de Psicología, 18 (2), 197-214. 


\section{CUESTIONARIO PARA LA EVALUACIÓN DE LOS VALORES SOCIOPERSONA-} LES PARA LA CONVIVEnCIA (De la Fuente, Peralta y Sánchez, 2006). Versión racional original (pensamientos-sentimientos-acciones).

Nombre y apellidos

Edad curso Centro de enseñanza Localidad

A continuación encontrarás distintas afirmaciones, por favor indica, tachando con un aspa $(\mathrm{X})$, en qué medida estás de acuerdo con ellas, sabiendo que 1= «Muy en desacuerdo», $2=\langle$ En desacuerdo», $3=$ «Regular de acuerdo», 4= «Bastante de acuerdo» y $5=$ «My de acuerdo»

1. Pienso que comportarse de forma amable es importante.

2. Estoy convencido que cuando ayudo a otras personas, sin esperar nada a cambio, es satisfactorio.

3. Considero que tengo bastantes amigos.

4. Pienso que admirar y valorar a los demás contribuye a mejorar mis relaciones con ellos.

5. Considero que para mí tiene mucha importancia aprender cosas en el Instituto.

6. Tengo muy claro que es bueno ser agradecido por los favores que te hacen.

7. Pienso que podría hacer cosas buenas por los demás que la mayoría de las personas no se atreven.

8. Considero que ser legal con los demás me hace ser mejor persona.

9. Sé que debo observar mi propia conducta, reflexionar sobre ella y concederme cosas agradables cuando actúo de forma apropiada.

10. Pienso que soy una persona bien valorada.

11. Reconozco que me gusta darle cosas personales mías a los demás.

12. Comprendo que antes de hacer cosas arriesgadas debo pensar en las consecuencias que me pueden acarrear.

13. Pienso que está mal hacerle sufrir o causarle daño a los demás.

14. Estoy seguro que la relación entre las personas es mejor cuando me esfuerzo por agradar a los demás.

15. Considero que es positivo arreglar los asuntos entre las personas dialogando.

16. Pienso que respetar a los demás evita problemas y nos hace la vida más agradable a todos. 
17. Creo que ser educado es una buena cualidad en las personas.

18. Pienso que las personas que tienen un comportamiento estable son mejor aceptadas por los demás.

19. Estoy seguro que esforzándome puedo conseguir lo que yo quiera.

20. Pienso que las personas que tienen éxito en la vida son mejor aceptadas por otras personas.

21. Pienso que cuando vienen personas de fuera debemos darles buena acogida.

22. Considero que soy una persona que presta ayuda a los demás.

23. Estoy convencido que cuando actúo con justicia evito problemas.

24. Reconozco que las normas y órdenes que nos dan los profesores y los adultos son para nuestro bien.

25. Pienso que si todos somos ordenados actuaremos mejor en la vida.

26. Considero que el respeto a profesores y compañeros favorece la convivencia.

27. Creo que los alumnos podemos sentirnos seguros ante cualquier peligro en los Institutos.

28. Pienso que los buenos modales mejoran las relaciones entre las personas.

29. Estoy seguro que soy una persona valiente.

30. Pienso que los más débiles o distintos por algo (sexo, raza, país de procedencia, etc) tienen mayor tendencia a ser perjudicados por otras personas.

31. Pienso que estoy preparado para enfrentarme a cualquier cosa.

32. Siento satisfacción interior cuando encuentro a personas amables.

33. Encuentro emociones agradables cuando, sin esperar nada a cambio, ayudo a los demás.

34. Me siento a gusto porque tengo amigos en quienes confiar.

35. Me siento bien cuando valoramos a otras personas.

36. Disfruto aprendiendo los contenidos de las asignaturas.

37. Me siento a gusto conmigo mismo cuando agradezco los favores que me hacen.

38. Me emociona ver que los demás me admiran porque hago cosas atrevidas.

39. Me siento mal cuando observo a personas que no son legales con los demás.

40. Me produce satisfacción cuando observo que acierto en las cosas que hago, reflexionando cómo las he logrado y reconociéndome mérito por ello.

41. Me siento agraciado cuando me valoran positivamente otras personas.

42. Me siento bien cuando tengo que dar cosas personales a los demás.

43. Me satisfecho cuando tengo que dar cosas personales a los demás.

44. Me entristezco cuando observo que hacen sufrir o padecer daño a otros. 
45. Me siento infeliz cuando veo que alguien tiene malas maneras o malos modales con los demás.

46. Me siento desdichado cuando alguien más poderoso que yo arregla las cosas por la fuerza y no con palabras.

47. Siento heridos mis sentimientos cuando observo que no respetan a los demás.

48. Me siento disgustado conmigo mismo cuando he actuado de mala manera con los demás.

49. Me agradan las personas que siempre sé como se van a comportar.

50. Me siento muy orgulloso de mí cuando consigo mis metas porque me esfuerzo.

51. Me siento muy feliz cuando consigo lo que me he propuesto.

52. Me siento satisfecho cuando llegan personas forasteras y las tratamos bien.

53. Me siento valioso cuando me piden ayuda los demás.

54. Me siento mal cuando veo que no he sido justo con las otras personas.

55. Me siento muy enfadado cuando tengo que cumplir las normas establecidas por el Instituto.

56. Me pone de mal humor observar desorden.

57. Me altera negativamente mi estado de ánimo observar que no respetan a alguien.

58. Siento confianza en los Institutos porque no corro peligro dentro de ellos.

59. Me siento muy mal cuando he sido mal educado con alguien.

60. Me desagrada observar que alguien tenga temor de hacer algo que los demás sí podemos hacer.

61. Me molesta que se metan con otros que sean inferiores.

62. Me siento orgulloso cuando me enfrento a los demás.

63. Soy una persona que se porta de forma amable con los demás.

64. Soy una persona que me gusta hacer favores a los demás, aunque no me los devuelvan.

65. Si me veo en apuros, sé que tengo alguien en quien confiar.

66. Acostumbro a expresar el valor y admiración que siento por otros.

67. Soy una persona que se esfuerza y presta interés por aprender los contenidos de las asignaturas.

68. Soy una persona que acostumbro a agradecer los favores y ayudas que me prestan.

69. Soy una persona que hago cosas atrevidas.

70. Soy una persona que frecuentemente actúa de forma legal con los demás.

71. Acostumbro, cuando observo que he hecho algo bien, a decirme: «Ánimo, merece la pena seguir esforzándome» 
72. Soy una persona a la que reconocen de forma frecuente ser buena persona.

73. Soy una persona que dona o entrega cosas personales a otros.

74. Tengo la costumbre de pensar las consecuencias de lo que voy a hacer antes de hacerlo.

75. Procuro ayudar y dar ánimo a los que me necesitan.

76. Acostumbro a tener buenos modales y palabras con los que me rodean.

77. Procuro arreglar los problemas que tengo con los demás hablando con ellos.

78. Actúo de acuerdo con esta frase: «No hago a los demás lo que no me gusta que me hagan a mí»».

79. Soy una persona que acostumbra a comportarse de forma educada.

80. Sé gobernar mi propia conducta evitando pasar sin motivos desde una alegría exagerada o una pena muy grande.

81. Tengo la costumbre de dedicarle el tiempo y el esfuerzo que haga falta para conseguir lo que me propongo.

82. Hago todo lo posible por conseguir mis metas.

83. Cuando llegan forasteros siempre procuro atenderlos bien.

84. Normalmente tengo por costumbre ayudar a quien me necesita.

85. Soy una persona que lucha contra las injusticias.

86. Soy una persona que cumple de buen grado las normas establecidas.

87. Procuro colaborar para que mis materiales y los del instituto estén ordenados.

88. Siempre respeto los derechos de mis compañeros.

89. Soy una persona que evita cualquier peligro para mí y para los demás.

90. Soy una persona que tiene buenos modales con los demás.

91. Soy una persona atrevida.

92. Soy una persona que procura respetar a los que son más débiles que yo.

93. Me gusta desafiar a los que me rodean. 